\title{
Network Non-neutrality Debate: An Economic Analysis ${ }^{\star}$
}

\author{
Eitan Altman ${ }^{\star \star}$, Arnaud Legout, and Yuedong Xu \\ INRIA Sophia Antipolis, 2004 Route des Lucioles, France \\ \{eitan.altman, arnaud.legout\}@inria.fr, yuedong.xu@gmail.com
}

\begin{abstract}
This paper studies economic utilities and quality of service (QoS) in a two-sided non-neutral market where Internet service providers (ISPs) charge content providers (CPs) for the content delivery. We propose new models that involve a CP, an ISP, end users and advertisers. The CP may have either a subscription revenue model (charging end users) or an advertisement revenue model (charging advertisers). We formulate the interactions between the ISP and the CP as a noncooperative game for the former and an optimization problem for the latter. Our analysis shows that the revenue model of the CP plays a significant role in a non-neutral Internet. With the subscription model, both the ISP and the CP receive better (or worse) utilities as well as QoS in the presence of the side payment at the same time. With the advertisement model, the side payment impedes the CP from investing on its contents.
\end{abstract}

Keywords: Network Non-neutrality, Side Payment, Nash Equilibrium, Bargaining.

\section{Introduction}

Network neutrality, one of the foundations of Internet, is commonly admitted that ISPs must not discriminate traffic in order to favor specific content providers [1. However, the principle of network neutrality has been challenged recently. The main reason is that new broadband applications cause huge amount of traffic without generating direct revenues for ISPs. Hence, ISPs want to get additional revenues from CPs that are not directly connected to them. For instance, a residential ISP might want to charge Youtube in order to give a premium quality of service to Youtube traffic. This kind of monetary flows, which violates the principle of network neutrality, are called two-sided payment. We use the term side payment to name the money charged by ISPs from CPs exclusively.

On the one hand, the opponents of network neutrality argue that it does not give any incentive for ISPs to invest in the infrastructure. This incentive issue is even more severe in two cases: the one of tier-one ISPs that support a high load, but do not get any revenue from CPs; and the one of $3 \mathrm{G}$ wireless networks that need to invest a huge amount of money to purchase spectrum. On the other

\footnotetext{
* The work was supported by the INRIA ARC Meneur on Network Neutrality.

${ }^{\star \star}$ Corresponding author.

J. Domingo-Pascual et al. (Eds.): NETWORKING 2011, Part II, LNCS 6641, pp. 68-81, 2011.

(C) IFIP International Federation for Information Processing 2011
} 
hand, advocates of network neutrality claim that violating it using side payment will lead to unbalanced revenues among ISPs and CPs, thus a market instability.

Recent work addressed the problem of network neutrality from various perspectives [23456789]. Among these work, [234] are the closest to our work. Musacchio et al. 2] compare one-sided and two-sided pricing of ISPs. However, they only investigate an example where the joint investments of CPs and ISPs bring revenue from advertisers to CPs. In [3], the authors show how side payment is harmful for all the parties involved such as ISP and CP. Altman et al. in 4] present an interesting bargaining framework to decide how much the ISP should charge the CP. However, their models might give a biased conclusion by overlooking the end users' sensitivity towards the prices of the CP and the ISP. Authors in [13] study a two sided model where a continuum of CPs obtains revenue either from the end users or from the advertisers. The ISP charges the CPs according to the qualities of connections chosen by them. Our work is different from 13 in two aspects. First, we consider the QoS provision of the ISP to the $\mathrm{CP}$ instead of the QoS differentiation among a continuum of CPs. Second, we formulate different mathematical models. The relative price sensitivity is considered in the subscription model and the investment of the CP is incorporated into the advertisement model.

In this paper, we unravel the conflicts of the side payment in a more general context. We consider a simplified market composed of one ISP, one CP, some advertisers, and a large number of end users. The ISP charges end-users based on their usage and sets their QoS level according to the price paid. The CP can either have a subscription based or an advertisement based revenue model. For the subscription based revenue model, the CP gets its revenue from the subscription paid by end-users. End-users adapt their demand of contents based on the price paid to the ISP and the CP. For the advertisement based revenue model, the CP gets its revenue from advertisers. End users adapt the demand according to the price paid to the ISP and the CP's investment on its contents.

Our work differs from related work 234 by: i) incorporating the QoS provided by the ISP, ii) studying different revenue models of the $\mathrm{CP}$, and iii) introducing the relative price sensitivity of end users in the subscription model. Especially, in the subscription model, the relative price sensitivity decides whether the side payment is beneficial (or harmful) to the ISP and the CP. Our finding contradicts the previous work (e.g. 3]) that argues that the side payment is harmful for all parties involved. In the advertisement model, the ability of the CP's investment to attract the traffic of end users plays a key role. It determines whether the side payment is profitable for the ISP and the CP. Our main contributions are the following.

- We present new features in the mathematical modeling that include the QoS, the relative price sensitivity of end-users, and the CP's revenue models.

- We analytically show that the side payment from the CP to the ISP is beneficial to the ISP and the CP in terms of profits under certain conditions.

- We utilize bargaining games based on 4 to investigate how the side payment is determined. 
The rest of this work is organized as follows. In section 2 we model the economic behaviors of ISP, CP, advertisers and end users. Section 3 and 4 study the impact of the side payment and its bargaining outcomes. Section 5 presents numerical study to validate our claims. Section [6 concludes this paper.

\section{Basic Model}

In this section, we first introduce the revenue models of the ISP and the CP. Then, we formulate a game problem and an optimization problem for the selfish ISP and the CP. Finally, we describe the bargaining games in a two-sided market.

\subsection{Revenue Models}

We consider a simplified networking market with four economic entities, namely the advertisers, the CP, the ISP and end users. All the end users can access the contents of the CP only through the network infrastructure provided by the ISP. The ISP collects subscription fees from end users. It sets two market parameters $\left(p^{s}, q\right)$ where $p^{s}$ is the non-negative price of per-unit of demand, and $q$ is the QoS measure (e.g. delay, loss or block probability). End users can decide whether to connect to the ISP or not, or how much traffic they will request, depending on the bandwidth price and the QoS. The CP usually has two revenue models, the user subscription and the advertisement from clicks of users. These two models, though sometimes coexisting with each other, are studied separately in this work for clarity. The CP and the ISP interact with each other in a way that depends on the CP's revenue models. In the subscription based model, the CP competes with the ISP by charging users a price $p^{c}$ per-unit of content within a finite time. End users respond to $p^{s}, p^{c}$ and $q$ by setting their demands elastically. Though $p^{c}$ has a different unit as $p^{s}$, it can be mapped from the price per content into the price per bps (i.e. dividing the price of a content by its size in a finite time). The price $p^{c}$ not only can stand for a financial disutility, but also can represent the combination of this disutility together with a cost per quality. Thus a higher price may be associated with some better quality (this quality would stand for parameters different from the parameter $q$ which we will introduce later). Without loss of generality, $p^{s}$ and $p^{c}$ can be positive or 0 . For the advertisement based model, instead of charging users directly, the CP attracts users' clicks on online advertisements. The more traffic demands the end users generate, the higher the CP's revenue.

To better understand network neutrality and non-neutrality, we describe the monetary flows among different components. The arrows in Figure 1 represent the recipients of money. A "neutral network" does not allow an ISP to charge a $\mathrm{CP}$ for which it is not a direct provider for sending information to this ISP's users. On the contrary, monetary flow from a CP to an ISP appears when "network neutrality" is violated. The ISP may charge the CP an additional amount of money that we denote by $f(D)=p^{t} D$ where $p^{t}$ is the price of per-unit of demand. We denote by $\delta \in[0,1]$ the tax rate of this transferred revenue imposed by the regulator or the government. 


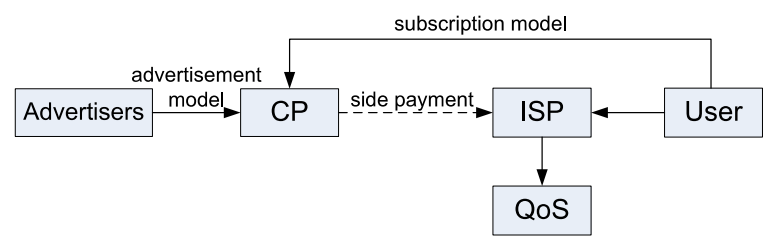

Fig. 1. Money flow of a non-neutral network

We present market demand functions for the subscription and the advertisement based revenue models.

Subscription model. Let us define the average demand of all users by $D$ that has

$$
D\left(p^{s}, p^{c}, q\right)=\max \left\{0, D_{0}-\alpha\left(p^{s}+\rho p^{c}\right)+\beta q\right\},
$$

where $D_{0}, \alpha, \beta$ and $\rho$ are all positive constants. The parameter $D_{0}$ reflects the total potential demand of users. The parameters $\alpha$ and $\beta$ denote the responsiveness of demand to the price and the QoS level of the ISP. The physical meaning of (1) can be interpreted in this way. When the prices of the ISP and the CP increase (resp. decrease), the demand decreases (resp. increases). If the QoS of the ISP is improved, the demand from users increases correspondingly. The parameter $\rho$ represents the relative sensitivity of $p^{c}$ to $p^{s}$. We deliberately set different sensitivities of end users to the prices of the ISP and the CP because $p^{c}$ and $p^{s}$ refers to different type of disutilities. If $\rho=1$, the prices of the ISP and the $\mathrm{CP}$ are regarded as having the same effect on $D$. When $\rho>1$, users are more sensitive to the change of $p^{c}$ than $p^{s}$. The positive prices $p^{s}$ and $p^{c}$ can not be arbitrarily high. They must guarantee a nonnegative demand $D$.

We denote $\mathcal{S}_{C P}$ to be the pricing strategy of the CP that has $\mathcal{S}_{\mathcal{C P}}=\left\{p^{c}\right.$ : $\left.p^{c} \geq 0\right\}$. The utility (or revenue equivalently) of the CP is expressed as

$$
U_{c p}=\left(p^{c}-p^{t}\right) D\left(p^{s}, p^{c}, q\right) .
$$

Note that the variable $D\left(p^{s}, p^{c}, q\right)$ is interchangeable with $D$ all the time. Next, we present the utility of the ISP with QoS consideration. We assume that the pricing strategy of the ISP is defined by $\mathcal{S}_{I S P}=\left\{\left(p^{s}, q\right): p^{s} \geq 0 ; 0<q \leq q_{\max }\right\}$. To sustain a certain QoS level of users, the ISP has to pay the costs for operating the backbone, the last-mile access, and the upgrade of the network, etc. Let $u(D, q)$ be the amount of bandwidth consumed by users that depends on the demand $D$ and the QoS level $q$. We assume that $u(D, q)$ is a positive, convex and strictly increasing function in the 2-tuple $(D, q)$. This is reasonable because a larger demand or higher QoS usually requires a larger bandwidth of the ISP. We now present a natural QoS metric as the expected delay1 1 . The expected delay is computed by the Kleinrock function that corresponds to the delay of $\mathrm{M} / \mathrm{M} / 1$ queue with FIFO discipline or $\mathrm{M} / \mathrm{G} / 1$ queue under processor sharing 10. Similar to [10, instead of using the actual delay, we consider the reciprocal

\footnotetext{
${ }^{1}$ The QoS metric can be the functions of packet loss rate or expected delay etc.
} 
of its square root, $q=\frac{1}{\sqrt{\text { Delay }}}=\sqrt{u(D, q)-D}$. Thus, the cost $C(D, q)$ can be expressed as $C(D, q)=p_{r} u(D, q)=p_{r} D+p_{r} q^{2}$, where $p^{r}$ the price of per-unit of bandwidth invested by the ISP. Therefore, the cost of the ISP is denoted by $C(D, q)=p^{r} u(D, q)$. The utility of the ISP is defined as the difference between revenue and cost:

$$
U_{i s p}=\left(p^{s}-p^{r}\right) D\left(p^{s}, p^{c}, q\right)+(1-\delta) p^{t} D\left(p^{s}, p^{c}, q\right)-p^{r} q^{2} .
$$

Advertisement model. Nowadays, a small proportion of CPs like Rapidshare and IPTV providers get their income from end users. Most of other CPs provide contents for free, but collect revenues from advertisers. The demand from users is transformed into attentions such as clicks or browsing of online advertisements. To attract more eyeballs, a CP needs to invest money on its contents, incurring a cost $c$. The investment improves the potential aggregate demand $D_{0}$ in return. Let $D_{0}(c)$ be a concave and strictly increasing function of the investment cost $c$. With abuse of notations we denote the strategy of the CP by $\mathcal{S}_{\mathcal{C P}}=\{c: c>0\}$. Hence, the demand to the CP and the ISP is written as

$$
D=D_{0}(c)-\alpha p^{s}+\beta q
$$

The utility of the ISP is the same as that in (3). Next, we describe the economic interaction between advertisers and the CP. There are $M$ advertisers interested in the $\mathrm{CP}$, each of which has a fixed budget $B$ in a given time interval (e.g., daily, weekly or monthly). An advertiser also has a valuation $v$ to declare its maximum willingness to pay for each attention. The valuation $v$ is a random variable in the range $[0, \bar{v}]$. Suppose that $v$ is characterized by probability density function (PDF) $x(v)$ and cumulative distribution function $(\mathrm{CDF}) X(v)$. We assume that the valuations of all advertisers are i.i.d. Let $p^{a}$ be the price of per attention charged by the CP. We denote by $D^{a}\left(p^{a}\right)$ the demand of attentions from advertisers to the CP. Therefore, $D^{a}$ can be expressed as 11.

$$
D^{a}=M B \cdot \operatorname{Prob}\left(v \geq p^{a}\right) / p^{a}=M B \cdot\left(1-X\left(p^{a}\right)\right) / p^{a} .
$$

When the CP increases $p^{a}$, the advertisers will reduce their purchase of attentions. It is also easy to see that the revenue of advertising, $p^{a} \cdot D^{a}$, decreases with regard to $p^{a}$ either. However, the attentions that the $\mathrm{CP}$ can provide is upper bounded by the demand of users through the ISP. Then, we can rewrite $D^{a}$ as that in [1] by

$$
D^{a}=\min \left\{D, \quad M B \cdot\left(1-X\left(p^{a}\right)\right) / p^{a}\right\} .
$$

Correspondingly, subtracting investment from revenue, we obtain the utility of the CP by

$$
U_{c p}=\left(p^{a}-p^{t}\right) D^{a}-c .
$$

Lemma 1. The optimal demand $D^{a}$ is a strictly decreasing function of $p^{a}$ if the pdf $x(v)$ is nonzero in $(0, \bar{v})$. 
The proofs of all lemmas and theorems can be found in [12. From (66), we can observe that the optimal price $p^{a *}$ is obtained at $D=M B \cdot\left(1-X\left(p^{a *}\right)\right) / p^{a *}$. Here, we denote a function $y(\cdot)$ such that $p^{a *}=y(D)$. According to the demand curve of attentions, $y(\cdot)$ is a decreasing function of $D$. The utility of the CP is a function of the demand $D$ and the $\operatorname{cost} c$, i.e. $U_{c p}=y(D) \cdot D-p^{t} D-c$.

\subsection{Problem Formulation}

With the subscription model, the strategy profile of the ISP is to set the 2tuple $\left(p^{s}, q\right)$ and that of the CP is to set $p^{c}$. This is actually a game problem in which the ISP and the CP compete by setting their prices. Since the ISP's QoS is tunable, we call this game "QoS Agile Price competition". With the advertisement model, the strategy of the ISP is still the price paid by end users, while that of the CP is to determine the investment level $c$. The ISP and the CP maximize their own utilities selfishly, but do not compete with each other. We name this maximization as "Strategic Pricing and Investment".

Definition 1. QoS Agile Price Competition In the subscription model, the $C P$ charges users based on their traffic demands. If the Nash equilibrium $N E^{(1)}=$ $\left\{p^{s *}, p^{c *}, q^{*}\right\}$ exists, it can be expressed as

$$
\begin{aligned}
\text { (G1) } U_{i s p}\left(p^{s *}, p^{c *}, q^{*}\right) & =\max _{\left\{p^{s}, q\right\} \in \mathcal{S}_{I S P}} U_{i s p}\left(p^{s}, p^{c *}, q\right), \\
U_{c p}\left(p^{s *}, p^{c *}, q^{*}\right) & =\max _{p^{c} \in \mathcal{S}_{C P}} U_{c p}\left(p^{s *}, p^{c}, q^{*}\right) .
\end{aligned}
$$

Definition 2. Strategic Pricing and Investment In the advertisement model, the ISP sets $\left(p^{s}, q\right)$ and the CP sets $c$ to optimize their individual utilities. If there exists an equilibrium $\left\{p^{s *}, q^{*}, c^{*}\right\}$, it can be solved by

$$
\text { (G2) } \begin{aligned}
U_{i s p}\left(p^{s *}, q^{*}, c^{*}\right) & =\max _{\left\{p^{s}, q\right\} \in \mathcal{S}_{I S P}} U_{i s p}\left(p^{s}, q, c^{*}\right), \\
U_{c p}\left(p^{s *}, q^{*}, c^{*}\right) & =\max _{c \in \mathcal{S}_{C P}} U_{c p}\left(p^{s *}, q^{*}, c\right) .
\end{aligned}
$$

\subsection{Bargaining Game}

The side payment serves as a fixed parameter in the above two problems. A subsequent and important problem is how large the side payment should be. When the ISP decides the side payment unilaterally, it might set a very high $p^{t}$ in order to obtain the best utility. However, this leads to a paradox when the ISP sets $p^{s}$ and $p^{t}$ at the meantime. With the subscription based model, if the ISP plays a strategy $\left(p^{t}, p^{s}, q\right)$ and the $\mathrm{CP}$ plays $p^{c}$, the noncooperative game leads to a zero demand and hence a zero income. This can be easily verified by taking the derivative of $U_{i s p}$ over $p^{t}$ (also see [3]). In other words, the ISP cannot set $p^{t}$ and $p^{s}$ simultaneously in the price competition. Similarly, with the advertisement based model, the ISP meets with the same paradox. There are two possible ways, the Stackelberg game and the bargaining game, to address this problem. Their basic principle is to let the ISP to choose $p^{t}$ and $p^{s}$ asynchronously. In this work, we consider the bargaining game in a market where the ISP and the CP 
usually have certain marketing powers. Our analysis in this work is close to the one presented in 4], but comes up with quite different observations.

We here analyze the bargaining games of the side payments that are played at different time sequences. The first one, namely pre-bargaining, models the situation that the bargaining takes place before the problems (G1) or (G2). The second one, defined as post-bargaining, models the occurrence of bargaining after the problems $(\mathbf{G 1})$ or $(\mathbf{G 2})$. Let $\gamma \in[0,1]$ be the bargaining power of the ISP over the CP. They negotiate the transfer price $p^{t}$ determined by $p^{t *}=$ $\arg \max _{p^{t}}\left(U_{i s p}\right)^{\gamma}\left(U_{c p}\right)^{1-\gamma}$. Since the utilities can only be positive, the optimal $p^{t}$ maximizes a virtual utility $U$

$$
p^{t *}=\arg \max _{p^{t}} U=\arg \max _{p^{t}}(1-\gamma) \log U_{c p}+\gamma \log U_{i s p} .
$$

We use (12) to find $p^{t}$ as a function of the strategies of the ISP and the CP.

\section{Price Competition of the Subscription Model}

In this section, we first investigate how the relative price sensitivity influences the price competition between the ISP and the CP. We then study the choice of the side payment under the framework of bargaining games.

\subsection{Properties of Price Competition}

This subsection investigates the impact of the side payment on Nash Equilibrium of the noncooperative game G1. Before eliciting the main result, we show some basic properties of the subscription based revenue model.

Lemma 2. The utility of the $C P, U_{c p}\left(p^{s}, p^{c}, q\right)$, in (2) is a finite, strictly concave function with regard to (w.r.t.) $p^{c}$.

Similarly, we draw the following conclusion.

Lemma 3. The utility of the ISP, $U_{i s p}\left(p^{s}, p^{c}, q\right)$, in (3) is a finite, strictly concave function w.r.t. the 2-tuple $\left(p^{s}, q\right)$ if the market parameters satisfy $4 \alpha p^{r}>\beta^{2}$.

For the QoS Agile Price Competition, we summarize our main results as below.

Lemma 4. When the ISP and the CP set their strategies selfishly,

- the Nash equilibrium $\left(p^{s *}, p^{c *}, q^{*}\right)$ is unique;

- the QoS level $q^{*}$ at the NE is influenced by the side payment in the ways:

- improved QoS with $\rho+\delta<1$;

- degraded $Q o S$ with $\rho+\delta>1$;

- unaffected $Q o S$ with $\rho+\delta=1$

if $\left(p^{s *}, p^{c *}, q^{*}\right)$ satisfy $p^{s *}>0, p^{c *}>0,0<q^{*}<q_{\max }$ and $4 \alpha p^{r}>\beta^{2}$. 
When the $\mathrm{NE}\left(p^{s *}, p^{c *}, q^{*}\right)$ is not at the boundary, we can yield the following expressions by solving the best response equations.

$$
\begin{aligned}
q^{*} & =\frac{\beta\left(D_{0}-\alpha p^{r}+\alpha p^{t}(1-\rho-\delta)\right)}{6 \alpha p^{r}-\beta^{2}}, \\
p^{c *} & =\frac{2 p^{r}\left(D_{0}-\alpha p^{r}+\alpha p^{t}(1-\rho-\delta)\right)}{\rho\left(6 \alpha p^{r}-\beta^{2}\right)}+p^{t}, \\
p^{s *} & =\frac{2 p^{r}\left(D_{0}-\alpha p^{r}+\alpha p^{t}(1-\rho-\delta)\right)}{6 \alpha p^{r}-\beta^{2}}+p^{r}-(1-\delta) p^{t}, \\
D^{*} & =\frac{2 p^{r} \alpha\left(D_{0}-\alpha p^{r}+\alpha p^{t}(1-\rho-\delta)\right)}{6 \alpha p^{r}-\beta^{2}},
\end{aligned}
$$

For the case that the NE is at the boundary, interested users can find it in the technical report 12 .

Lemma 4 means that the QoS provision of the ISP is influenced by the side payment. We interpret the results by considering $\rho$ and $\delta$ separately. When users are indifferent to the price set by the ISP and that by the CP (i.e., $\rho=1$ ), a positive tax rate $\delta$ leads to the degradation of $q$ in the presence of the side payment. Next, we let $\delta$ be 0 and investigate the impact of $\rho$. If users are more sensitive to the price of the ISP (i.e. $\rho<1$ ), the side payment is an incentive of the ISP to improve its QoS. Otherwise, charging side payment leads to an even poorer QoS of the ISP. Therefore, if users are more sensitive to the CP's price, a good strategy of the ISP is to share its revenue with the CP so that the latter sets a lower subscription fee.

\subsection{Bargaining of the Side Payment}

To highlight the bargaining of the side payment, we make the following two simplifications: i) the tax ratio $\delta$ is 0 , and ii) $p^{t}$ can be positive, zero or negative. We let $\delta=0$ because it turns out to have the similar effect as $\rho$. We have shown that a negative $p^{t}$ might benefit both the ISP and the CP in some situations. Hence, we do not require $p^{t}$ to be positive in the bargaining game. When $q$ reaches $q_{\max }$, the QoS is reflected as a fixed parameter in the demand model. To avoid considering the boundary cases of $q$, we also remove the constraint $q \leq q_{\max }$.

Pre-bargaining: In the pre-bargaining, $p^{t}$ is chosen based on the NE of the ISP and the CP. The equations (13) (15) yield the expression of $U$

$$
U=4 \log \left(D_{0}-\alpha p^{r}+\alpha p^{t}(1-\rho)\right)+\text { constant } .
$$

The utility $U$ is increasing or decreasing in $p^{t}$ depending on the sign of $(1-\rho)$. If $\rho<1$, a positive $p^{t}$ improves not only the QoS level of the ISP, but also the utilities of the ISP and the CP. As $p^{t}$ increases, $p^{s}$ decreases and $p^{c}$ increases consequently until $p^{s}$ hits 0 . Hence, in the pre-bargaining, $p^{s *}=0$. The prices $p^{t *}$ and $p^{c *}$ are computed by

$$
\begin{aligned}
p^{t *} & =\frac{p^{r}\left(4 \alpha p^{r}+2 D_{0}-\beta^{2}\right)}{4 \alpha p^{r}+2 \rho \alpha p^{r}-\beta^{2}} . \\
p^{c *} & =\frac{2 p^{r}\left(D_{0}-\alpha p^{r}+\alpha p^{t *}(1-\rho)\right)}{\rho\left(6 \alpha p^{r}-\beta^{2}\right)}+p^{t *} .
\end{aligned}
$$


On the contrary, when $\rho>1$, a negative $p^{t}$ benefits both of them. Then, $p^{t *}$ is a negative value such that $p^{c *}$ is 0 . When $\rho=1$, the QoS and the utilities are unaffected by any $p^{t}$. Among all these cases, the selection of $p^{t}$ is uninfluenced by the bargaining power $\gamma$.

Post-bargaining: For the post-bargaining, the ISP and the CP compete for the subscription of users first, knowing that they will bargain over $p^{t}$ afterwards 44. In brief, we find $p^{t}$ as a function of $p^{s}, p^{c}$ and $q$ first. Then, the ISP and the $\mathrm{CP}$ compete with each other by setting the prices. To solve the maximization in (12), we let $\frac{d U}{d p^{t}}$ be 0 and obtain

$$
p^{t}=\gamma p^{c}-(1-\gamma)\left(p^{s}-p^{r}\right)+(1-\gamma) p^{r} q^{2} / D .
$$

Submitting (20) to $U_{i s p}$, we rewrite the ISP's utility by

$$
U_{i s p}=\gamma\left(\left(p^{s}+p^{c}-p^{r}\right)\left(D_{0}-\alpha\left(p^{s}+\rho p^{c}\right)+\beta q\right)-p^{r} q^{2}\right) .
$$

The utility of the CP is proportional to that of the ISP, i.e. $\frac{U_{i s p}}{\gamma}=\frac{U_{c p}}{1-\gamma}$. After knowing $p^{t}$, we compute the derivatives $\frac{d U_{i s p}}{d p^{s}}, \frac{d U_{i s p}}{d q}$ and $\frac{d U_{c p}}{d p^{c}}$ by

$$
\begin{aligned}
& \frac{d U_{i s p}}{d p^{s}}=\gamma\left(D-\alpha\left(p^{s}+p^{c}-p^{r}\right)\right), \\
& \frac{d U_{i s p}}{d q}=\gamma\left(\beta\left(p^{s}+p^{c}-p^{r}\right)-2 p^{r} q\right), \\
& \frac{d U_{c p}}{d p^{c}}=(1-\gamma)\left(D-\alpha \rho\left(p^{s}+p^{c}-p^{r}\right)\right) .
\end{aligned}
$$

The best responses of $U_{i s p}$ and $U_{c p}$ will not happen at the same time unless $\rho=1$ or $p^{s}+p^{c}-p^{r}=0$. The condition $p^{s}+p^{c}-p^{r}=0$ does not hold because it leads to a zero demand $D$ and zero utilities. When $\rho$ is not 1 , only one of (22) and (24) is 0 . Here, we consider the case $\rho>1$. The utility $U_{c p}$ reaches its maximum upon $D=\alpha \rho\left(p^{s}+p^{c}-p^{r}\right)$, while $U_{i s p}$ is still strictly increasing w.r.t. $p^{s}$. Thus, the ISP increases $p^{s}$ until the demand $D$ is 0 , which contradicts the condition of a nonzero $D$. If $D=\alpha\left(p^{s}+p^{c}-p^{r}\right), \frac{d U_{c p}}{d p^{c}}$ is negative and $\frac{d U_{i s p}}{d p^{s}}$ is 0 . Then, the CP decreases $p^{c}$ until 0 and the ISP sets $p^{s}$ to achieve its best utility accordingly. By letting (24) be 0 , we can find $\left(p^{s}, q\right)$ at the Nash equilibrium

$$
q^{*}=\frac{\beta\left(D_{0}-\alpha p^{r}\right)}{4 \alpha p^{r}-\beta^{2}} \quad \text { and } \quad p^{s *}=\frac{2 p^{r}\left(D_{0}-\alpha p^{r}\right)}{4 \alpha p^{r}-\beta^{2}}+p^{r} .
$$

The price of the side payment, $p^{t *}$, is thus computed by

$$
p^{t}=-(1-\gamma) \frac{D_{0}-\alpha p^{r}}{2 \alpha} .
$$

When $\rho=1, p^{s *}$ and $p^{c *}$ can be arbitrary values in their feasible region that satisfy $p^{s *}+p^{c *}=p^{r}+\frac{2 p^{r}\left(D_{0}-\alpha p^{r}\right)}{4 p^{r} \alpha-\beta^{2}}$. Similar result has been shown in [4]. The analysis of $\rho<1$ is omitted here since it can be conducted in the same way. 


\section{Price, QoS and Investment Settings of the Advertisement Model}

This subsection analyzes how the side payment influences the optimal strategies of ISP and CP with the advertisement model. The bargaining games are adopted to determine the amount of the side payment. Compared with subscription based model, the advertisement based model exhibits quite different behaviors.

\subsection{Properties of the Advertisement Mode}

In general, the subscription model is limited to file storage CDNs, newspaper corporations, or big content owners such as movie producers. Most of CPs are not able to provide enough unique contents so that they do not charge users, but make money from online advertisements. In this subsection, we present the general properties of the advertisement model and a couple of case studies.

Lemma 5. For any feasible investment $c$ of the $C P$, there exists a best strategy of the ISP, $\left(p^{s}, q\right)$. When $c$ increases, the price and the QoS (i.e. $p^{s}$ and q) become larger.

In G2, the CP and the ISP do not compete with each other. On one hand, the ISP sets the two-tuple $\left(p^{s}, q\right)$ with the observation of $c$. On the other hand, the $\mathrm{CP}$ adjusts $c$ based on $\left(p^{s}, q\right)$. The investment of the CP brings more demand of end users, which increases the revenues of not only the ISP, but also the CP. Hence, different from G1, the problem G2 is not a game. Instead of studying the NE, we look into the optimal strategies of the ISP and the CP in G2.

Theorem 1. There exists a unique best strategy, namely $\left(p^{s *}, q^{*}, c^{*}\right)$, with the advertisement model if the revenue of the $C P, D \cdot y(D)$, is a concave function w.r.t. $D \geq 0$.

Lemma 6. The side payment from the CP to the ISP leads to a decreased investment on the contents when the best strategy $\left(p^{s *}, q^{*}, c^{*}\right)$ has $p^{s *}>0, q^{*}>0$ and $c^{*}>0$.

\subsection{Case Study}

In this subsection, we aim to find the best strategies of the ISP and the CP when the valuation of advertisers follows a uniform distribution or a normal distribution. Due to the page limit, the case of normal distribution is put in the technical report [12].

Recall that the potential aggregate demand of users, $D_{0}(c)$, is strictly increasing and concave w.r.t $c$. When the CP invests money on contents, $D_{0}$ becomes larger, while its growth rate shrinks. Here, we assume a log function of $D_{0}(c)$,

$$
D_{0}(c)=D_{0}^{0}+K \log (1+c)
$$


where the constant $K$ denotes the ability that the CP's investment brings the demand. The nonnegative constant $D_{0}^{0}$ denotes the potential aggregate demand of end users when $c$ is zero (the $\mathrm{CP}$ only provides free or basic contents). The utility of the ISP remains unchanged.

Uniform Distribution: Suppose $v$ follows a uniform distribution in the range $[0, \bar{v}]$. Then, the CDF $X\left(p^{a}\right)$ is expressed as $\frac{p^{a}}{\bar{v}}$. The optimal price $p^{a}$ is obtained when $D=\frac{M B}{p^{a}} \cdot\left(1-\frac{p^{a}}{\bar{v}}\right)$ in the range $[0, \bar{v}]$ (see subsection 2.1). Alternatively, there has

$$
p^{a}=\frac{M B \bar{v}}{M B+D \bar{v}}
$$

The above expressions yield the utility of the CP by

$$
U_{c p}=\frac{M B \bar{v} D}{M B+D \bar{v}}-c-p^{t} D .
$$

Deriving $U_{c p}$ over $c$, we obtain

$$
\frac{d U_{c p}}{d c}=\left(\frac{(M B)^{2} \bar{v}}{(M B+D \bar{v})^{2}}-p^{t}\right) \cdot \frac{K}{1+c}-1 .
$$

We let (29) be 0 and get

$$
c=K\left(\frac{(M B)^{2} \bar{v}}{(M B+D \bar{v})^{2}}-p^{t}\right)-1 .
$$

The rule of the ISP to decide $\left(p^{s}, q\right)$ is the same as that in the subscription model, except that the aggregate demand is not a constant, but a function of $c$,

$$
c=\exp \left(\frac{\frac{D}{2 p^{r} \alpha}\left(4 p^{r} \alpha-\beta^{2}\right)-D_{0}^{0}+\alpha p^{r}-(1-\delta) p^{t} \alpha}{K}\right)-1 .
$$

Note that (30) is strictly decreasing and (31) is strictly increasing w.r.t. D. They constitute a fixed-point equation for the 2-tuple $\left(D^{*}, c^{*}\right)$. In the beginning, we assume that the optimal strategies are not on the boundary. When $D$ approaches infinity, (30) is negative while (31) is positive. When $D$ is zero, if (30) is larger than (31), there exists a unique fixed-point solution. In this fixed point, the ISP and the CP cannot benefit from changing their strategy unilaterally. We can solve $c^{*}$ and $D^{*}$ numerically using a binary search. If (30) is smaller than (31) when $D$ is 0 , the best strategy of the CP is exactly $c=0$. The physical interpretation is that the increased revenue from advertisers cannot compensate the investment on the contents. Once $D^{*}$ and $c^{*}$ are derived, we can solve $p^{s *}$ and $q^{*}$ subsequently. In this fixed-point equation, $p^{t}$ greatly influences the optimal investment $c^{*}$. When $p^{t}$ grows, the right sides of (30) and (31) decrease at the mean time. The crossing point of two curves, (30) and (31), shifts toward the direction of smaller $c$ as shown in Lemma 6. Intuitively, the contents of the CP become less when the ISP charges a positive $p^{t}$. The boundary case of the optimal strategies as well as the bargaining games over $p^{t}$ are analyzed in $[12$. 


\section{Evaluation}

We present some numerical results to reveal how the QoS, prices of the ISP and the CP, as well as their utilities evolve when the price of the side payment changes. The impact of bargaining power on the side payment is also illustrated. More numerical examples are demonstrated in the technical report [12].

Subscription Model: We consider a networking market where the demand function is given by $D=200-10\left(p^{s}+\rho p^{c}\right)+0.5 q$. The operational cost of per-unit of bandwidth is set to $p^{r}=1$. Two situations, $\rho=0.5$ and $\rho=1.5$, are evaluated. The tax rate $\delta$ is set to 0 for simplicity. As is analyzed, the side payment benefits the ISP and the CP depending on whether $\rho$ is greater than 1 or not. In figure 2, $p^{t}$ has different impacts on utilities of the ISP and the CP. When $\rho>1$, end users are more sensitive to the change of $p^{c}$ than $p^{s}$. A positive $p^{t}$ leads to the increase of $p^{c}$, causing a tremendous decrease of demand. Hence, both the ISP and the CP lose revenues w.r.t. a positive $p^{t}$. Figure 3 further shows that a positive $p^{t}$ yields a better $\operatorname{QoS}$ if $\rho<1$ and a worse QoS if $\rho>1$.

Next, the ISP and the CP bargain with each other to determine $p^{t}$. We relax the choice of $p^{t}$ so that it can be negative. In the pre-bargaining game, $p^{t}$ is independent of the bargaining power $\gamma$. The optimal $p^{t}$ is obtained when $p^{s *}$ decreases to 0 , its lower bound. We evaluate $p^{t}$ by changing $\rho$ and $\alpha$ in figure 4 . When $\rho$ increases from 0.2 to $2, p^{t}$ decreases until it becomes negative. A negative $p^{t}$ means that the ISP needs to transfer revenue to the CP instead. When $\rho=1$, $p^{t}$ can be an arbitrary value as long as $p^{s *}$ and $p^{c *}$ are nonnegative. Figure 4 also shows that a larger $\alpha$ results in a smaller absolute value of $p^{t}$.
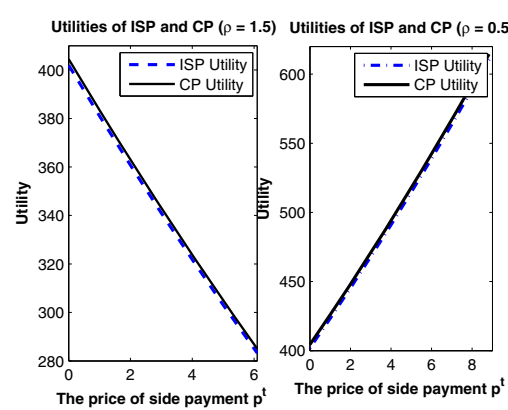

Fig. 2. Subscription Model: Utilities of the ISP and the CP

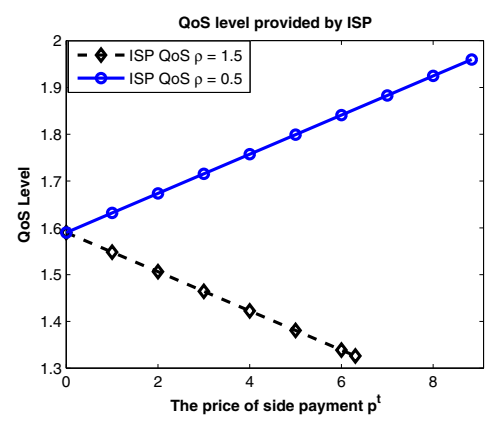

Fig. 3. Subscription Model: The QoS level provided by the ISP

Advertisement Model: In the advertisement model, we consider the demand function $D=K \log (1+c)-10 p^{s}+0.5 q$. The coefficient $K$ reflects the efficiency of the CP's investment to attract end users. The valuation of each click/browsing follows uniform distribution in the range $[0,10]$. The total budget of advertisers is set to 1000 . We conduct two sets of experiments. The first one is to evaluate 


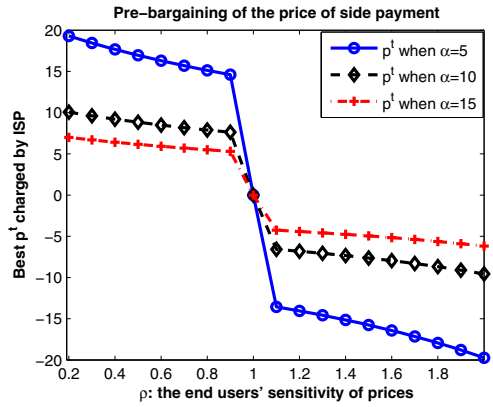

Fig. 4. Subscription Model: Prebargaining of $p^{t}$

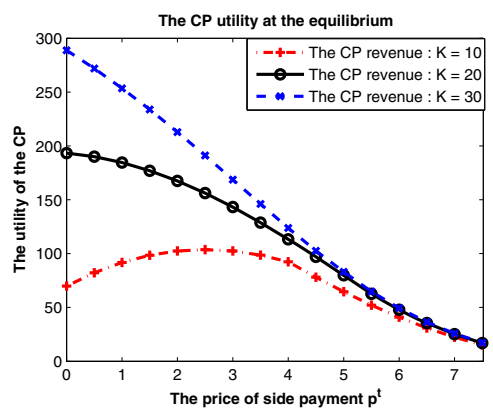

Fig. 6. Advertisement Model: The ISP's utility at the equilibrium

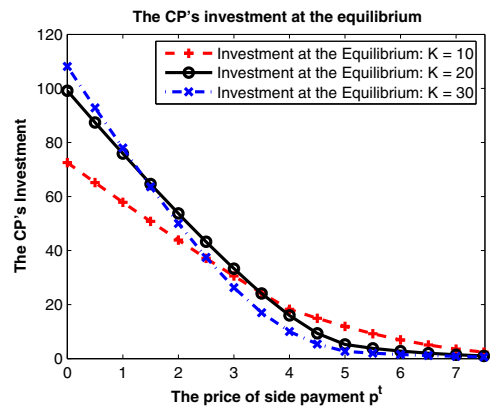

Fig. 5. Advertisement Model: CP's investment at the equilibrium

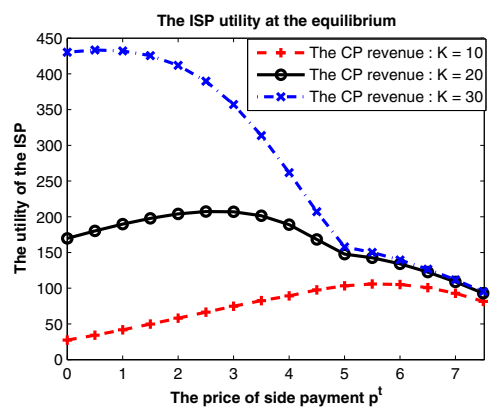

Fig. 7. Advertisement Model: The ISP's utility at the equilibrium

the impact of the side payment on the best strategies of the ISP and the CP. The second one is to find the optimal $p^{t}$ in the pre-bargaining game. In figure 5. the CP's investment is a decreasing function of $p^{t}$. When $p^{t}$ is large enough, $c$ reduces to 0 . Figure 6 illustrates the utility of the $\mathrm{CP}$ when $p^{t}$ and $K$ change. The CP's utility increases first and then decreases with $K=10$ when $p^{t}$ increases. For the cases $K=20$ and 30, the increase of $p^{t}$ usually leads to the decrease of revenues. In figure 7 , the utility of the ISP with $K=10$ and 20 increases first and then decreases when $p^{t}$ grows. These curves present important insights on the interaction between the CP and the ISP. If the contents invested by the CP can bring a large demand, the side payment is not good for both the ISP and the CP. On the contrary, when the efficiency $K$ is small, the CP can obtain more utility by paying money to the ISP.

\section{Conclusion and Future Work}

In this paper, we first answer under what situations the side payment charged by the ISP is beneficial for the ISP (or the CP). Then, we study how the price 
of the side payment is determined. Our models take account of three important features, the relative price sensitivity, the CP's revenue models, and the QoS provided by the ISP. With the subscription model, the relative price sensitivity determines whether the ISP should charge the side payment from the CP or not. With the advertisement model, the charge of the side payment depends on the ability of the CP's investment to attract the demand.

\section{References}

1. Hahn, R., Wallsten, S.: The economics of net neutrality. The Berkeley Economic Press Economists Voice 3(6), 1-7 (2006)

2. Musacchio, J., Schwartz, G., Walrand, J.: A two-sided market analysis of provider investment incentives with an application to the net-neutrality issue. Review of Network Economics 8(1) (2009)

3. Altman, E., Bernhard, P., Caron, S., Kesidis, G., Rojas-Mora, J., Wong, S.L.: A Study of Non-Neutral Networks with Usage-based Prices. In: 3rd ETM Workshop of ITC Conference (2010)

4. Altman, E., Hanawal, M.K., Sundaresan, R.: Nonneutral network and the role of bargaining power in side payments. In: NetCoop, Ghent, Belgium (November 2010)

5. Economides, N., Tag, J.: Net neutrality on the internet: A two-sided market analysis, working paper, http://ideas.repec.org/p/net/wpaper/0714.html

6. Claudia Saavedra, V.: Bargaining power and the net neutrality debate, working paper (2010), http://sites.google.com/site/claudiasaavedra/

7. Choi, J.P., Kim, B.C.: Net Neutrality and Investment Incentives, working paper (2008), http://ideas.repec.org/p/ces/ceswps/_2390.html

8. Ma, T.B., Chiu, D.M., Lui, J.C.S., et al.: On Cooperative Settlement Between Content, Transit and Eyeball Internet Service Providers. To appear in IEEE/ACM Trans. on Networking

9. Bangera, P., Gorinsky, S.: Impact of Prefix Hijacking on Payments of Providers. In: Proc. of COMSNETS 2011 (January 2011)

10. El-Azouzi, R., Altman, E., Wynter, L.: Telecommunications Network Equilibrium with Price and Quality-of-Service Characteristics. In: Proc. of ITC (2003)

11. Liu, J., Chiu, D.M.: Mathematical Modeling of Competition in Sponsored Search Market. In: ACM Workshop on NetEcon 2010, Vancouver (2010)

12. Altman, E., Legout, A., Xu, Y.D.: Network Non-neutrality Debate: An Economic Analysis, in Technical Report (2010), http://arxiv.org/abs/1012.5862

13. Hermalin, B.E., Katz, M.L.: The Economics of Product-Line Restrictions With an Application to the Network Neutrality Debate. Information Economics and Policy 19, 215-248 (2007) 